\title{
Correção endovascular de pseudoaneurisma de tronco tibiofibular secundário a endocardite - relato de caso
}

\section{Endocarditis-related tibioperoneal trunk pseudoaneurysm managed with endovascular treatment - case report}

Marcio Miyamotto ${ }^{1,2,3}$, Gabriele Simões Marcusso ${ }^{1,2,4}$, Thalita Toldo Ferreira ${ }^{1,2,4}$, Matheus Schimidt Evangelista ${ }^{1,2,4}$, Giana Caroline Strack Neves ${ }^{3}$, lan Gimenez Ribeiro ${ }^{3}$, Cintia Lopes Raymundo ${ }^{2,3}$, Fernanda Reis Gomes ${ }^{5}$

\begin{abstract}
Resumo
Os aneurismas do tronco tibiofibular são raros e consistem principalmente em pseudoaneurismas. Os autores descrevem um caso incomum de pseudoaneurisma do tronco tibiofibular secundário a endocardite bacteriana diagnosticada e tratada vários anos antes. Após a exclusão de um processo infeccioso ativo, o paciente foi tratado com sucesso através do implante percutâneo de um stent recoberto. O tratamento endovascular, neste contexto, apresentou uma alternativa segura e eficaz.
\end{abstract}

Palavras-chave: pseudoaneurisma; tronco tibiofibular; endocardite; endovascular.

\begin{abstract}
Tibioperoneal trunk aneurysms are rare and the majority of them are pseudoaneurysms This report describes an unusual case of a pseudoaneurysm secondary to bacterial endocarditis diagnosed and treated several years previously. After ruling out ongoing infection, the patient was successfully treated by percutaneous covered stent implantation. In this scenario, the use of endovascular techniques offered a safe and effective alternative treatment.
\end{abstract}

Keywords: pseudoaneurysm; tibioperoneal trunk; endocarditis; endovascular

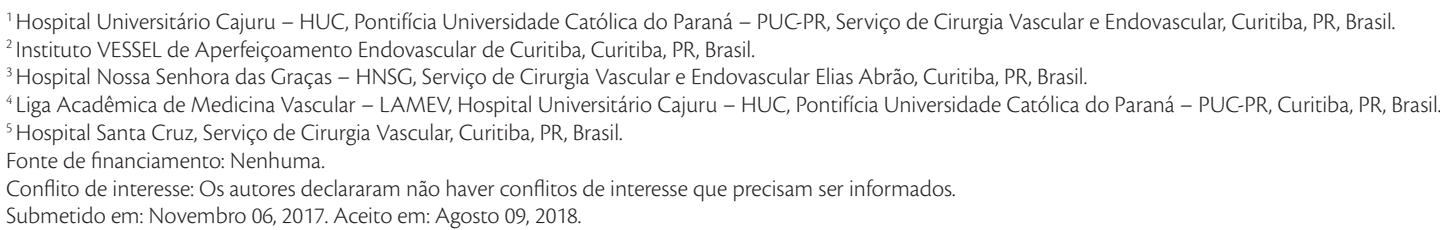




\section{INTRODUÇÃO}

O termo aneurisma micótico foi utilizado pela primeira vez por Sir William Osler, em 1885, em sua dissertação sobre endocardite bacteriana. Naquela ocasião, foi identificada a ocorrência de embolização séptica distal em até $80 \%$ dos casos, a qual seria responsável pela formação desses pseudoaneurismas ${ }^{1}$. Esse fato pode ser comprovado pela súbita diminuição da incidência dessa complicação após o advento da antibioticoterapia e das técnicas de substituição das válvulas cardíacas infectadas no tratamento das endocardites ${ }^{2}$.

A maioria dos casos de embolização arterial distal relacionada à endocardite ocorre na bifurcação da artéria femoral comum ${ }^{2}$. Poucos casos foram descritos envolvendo as artérias infrageniculares ${ }^{2-7}$. No presente artigo, relatamos um caso de pseudoaneurisma de tronco tibiofibular com apresentação tardia ao tratamento de endocardite bacteriana. Nesse caso, após exclusão de qualquer processo infeccioso ativo, optamos pelo implante de um stent recoberto como forma de tratamento.

\section{DESCRIÇÃO DO CASO}

Paciente do sexo masculino, 65 anos, apresentou queixa de aumento de volume na face posterior do terço proximal da perna esquerda. Possuía história pregressa de internamento prolongado para tratamento de endocardite bacteriana, sendo realizadas, nessa ocasião, duas cirurgias para troca valvar mitral. Apresentava também duas cirurgias abdominais prévias por tumor intestinal e uma cirurgia de varizes.

Ao exame físico, apresentava uma massa pulsátil na região posterior do terço proximal da perna esquerda. Os pulsos femoral, poplíteo e dorsal do pé de ambos os membros inferiores eram palpáveis e simétricos. O pulso da artéria tibial posterior do membro inferior esquerdo era impalpável, enquanto o pulso da artéria tibial posterior do membro inferior direito era palpável.

Foi solicitada uma angiorressonância, que evidenciou a presença de uma dilatação de formato sacular, medindo 4,4 cm em seu maior diâmetro, no tronco tibiofibular do membro inferior esquerdo, ao nível da origem da artéria tibial posterior. A artéria tibial posterior encontrava-se ocluída (Figura 1). Para investigação complementar, foram solicitados exames laboratoriais (provas de atividade inflamatória, coagulograma e hemograma completo), que apresentaram resultados normais, além de hemoculturas, que foram negativas.

Afastadas outras prováveis etiologias ou infecção ativa devido ao histórico de endocardite bacteriana, optou-se por um tratamento menos invasivo considerando a fisiopatologia inflamatória/infecciosa do processo,

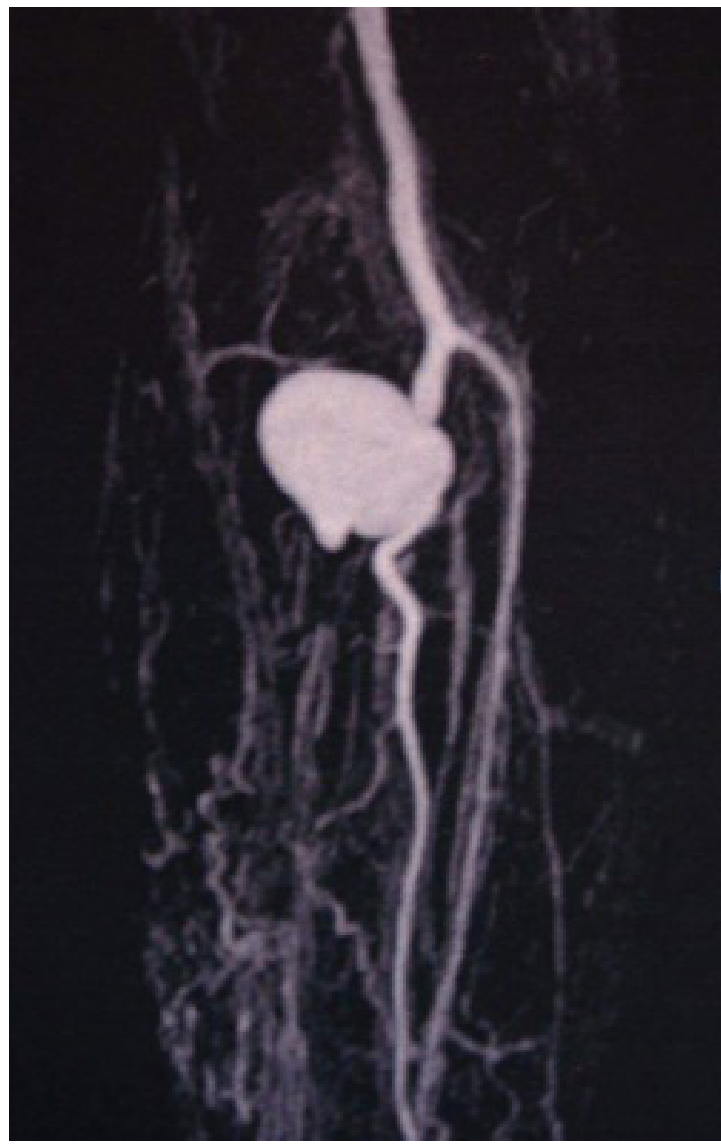

Figura 1. Angiorressonância de membro inferior esquerdo evidenciando pseudoaneurisma em tronco tibiofibular e oclusão da artéria tibial posterior.

o tamanho e a localização do pseudoaneurisma. O paciente foi então submetido ao implante de um stent recoberto autoexpansível (Gore VIABAHN $6.0 \times 50 \mathrm{~mm})$ no tronco tibiofibular, mantendo a perviedade da artéria fibular e excluindo o aneurisma. Devido à presença do pseudoaneurisma, a transição entre o tronco tibiofibular e a artéria fibular encontrava-se ectasiada, minimizando a dificuldade relacionada à redução de diâmetro distal. A angiorressonância de controle evidenciou a ausência de perfusão do saco aneurismático, além da perviedade da artéria fibular (Figura 2).

O paciente foi seguido por um período de 10 anos após o tratamento, não apresentando recorrência dos sintomas durante o período e mantendo índice tornozelo-braquial (ITB) de 1,0.

\section{DISCUSSÃO}

Os aneurismas periféricos de localização infrapoplítea são raros e, quando ocorrem, geralmente são pseudoaneurismas. Existem poucos casos descritos 


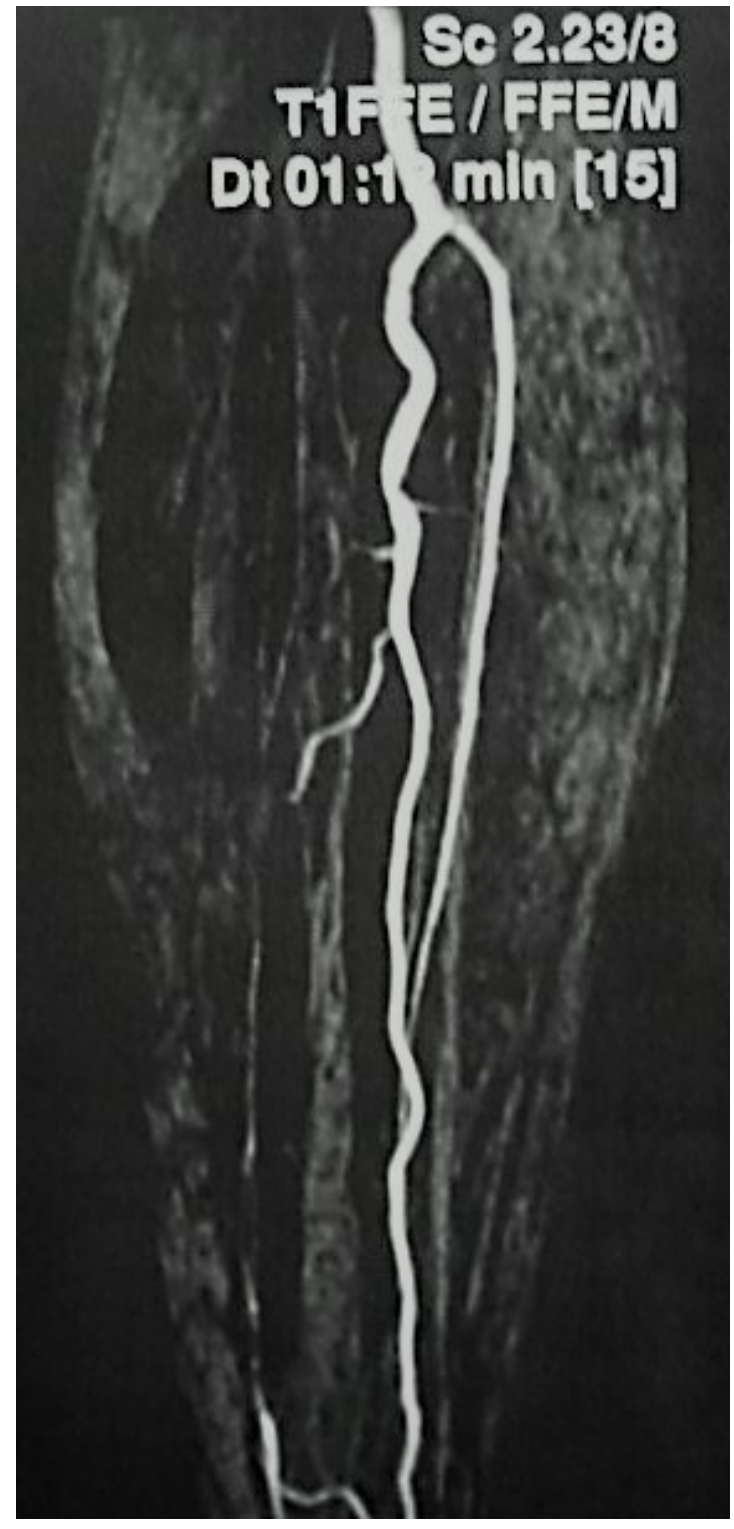

Figura 2. Angiorressonância de controle evidenciando ausência de perfusão do saco aneurismático e perviedade da artéria fibular.

na literatura, sendo comumente relacionados a traumatismos arteriais (como fraturas tibiais ou maleolares), acessos venosos periféricos, angioplastia transluminal percutânea ou embolizações secundárias a endocardite bacteriana ${ }^{3}$. Estes são ainda mais raros devido ao uso de antibióticos e procedimentos cirúrgicos precoces no tratamento da endocardite bacteriana e, quando ocorrem, costumam acometer mais comumente artérias axiais como a aorta, artérias cerebrais, artérias mesentéricas e a artéria femoral comum ${ }^{4}$.

Os pseudoaneurismas micóticos podem se desenvolver após a infecção do vaso ou podem ser resultado da infecção secundária de um aneurisma pré-existente.
Ambos são raros e apresentam uma incidência entre 1 e 3,7\%, considerando os aneurismas de configuração sacular ${ }^{7}$. Ao contrário do que o nome sugere, os aneurismas micóticos geralmente são secundários a uma infecção bacteriana, mais comumente por Staphylococcus aureus e Salmonella spp. Também podem ocorrer como consequência de infecção por fungos, embora seja muito incomum, ocorrendo em 1 a $2 \%$ dos $\operatorname{casos}^{3,7}$.

A fisiopatogenia do pseudoaneurisma micótico está relacionada a microembolizações sépticas que acometem os vasa vasorum, levando a isquemia, necrose focal e fragilidade da parede arterial, resultando em ruptura e formação do pseudoaneurisma. Na maioria dos relatos na literatura, tais embolizações geram pseudoaneurismas em vasos axiais como aorta, artérias mesentéricas, vasos cerebrais e artéria femoral, raramente atingindo o território infrapoplíteo, sendo que existiam apenas 10 casos descritos na literatura até $2012^{2,4-7}$.

A apresentação clínica é variável, podendo o paciente ser assintomático ou apresentar queixas de edema, dor e sensação de massa pulsátil na região acometida. Os sintomas relacionados à compressão nervosa e venosa são dependentes do tamanho e da localização do pseudoaneurisma. A ruptura e a embolização distal são complicações de extrema urgência e gravidade, agravadas ainda mais quando há presença de processo infeccioso ativo ${ }^{8}$.

$\mathrm{O}$ reconhecimento e o tratamento precoce do pseudoaneurisma micótico são essenciais para a prevenção de complicações, cujo manejo deve ser individualizado a cada caso. Tradicionalmente, o tratamento consiste na ressecção do pseudoaneurisma com debridamento dos tecidos necróticos e infectados, ligadura do vaso acometido e revascularização do membro, que deve ser realizada preferencialmente com enxerto autógeno, por via anatômica ou extra-anatômica, seguida de um período prolongado de antibioticoterapia ${ }^{6}$. Em 1992, Donald et al. relataram um caso de pseudoaneurisma micótico em tronco tibiofibular que surgiu 18 meses após um quadro de endocardite bacteriana por Streptococcus viridans e foi manejado com sucesso por reparo aberto, com restituição da circulação arterial distal ${ }^{6}$. Após isso, outros casos de pseudoaneurisma nessa topografia também foram relatados na literatura, mantendo o manejo com a técnica cirúrgica tradicional como primeira escolha ${ }^{2-7}$.

O uso de técnicas endovasculares para tratamento de pseudoaneurismas periféricos se iniciou em 1994. Geremia et al. implantaram stents endovasculares em pseudoaneurismas induzidos em artérias carótidas de 
cães e observaram trombose do saco do pseudoaneurisma e perviedade do vaso acometido?.

Entretanto, o uso da técnica endovascular para o tratamento de pseudoaneurismas infrapoplíteos possui poucos relatos na literatura. Sadat et al. realizaram embolização com molas em um caso de pseudoaneurisma de artéria fibular pós-embolectomia com cateter de Fogarty ${ }^{8}$. Houve oclusão total do fluxo no pseudoaneurisma, e o paciente recebeu alta hospitalar assintomático, dois dias após o procedimento ${ }^{8}$. Larena-Avellaneda et al. também realizaram a embolização com molas para o tratamento de um paciente com pseudoaneurisma micótico após endocardite por Candida albicans ${ }^{7}$. No caso aqui relatado, foi utilizada a técnica endovascular com colocação de stent revestido flexível para exclusão do pseudoaneurisma, sendo que não foram encontrados casos semelhantes na literatura. A apresentação tardia do pseudoaneurisma contribuiu para que fosse possível a utilização dessa técnica, já que não havia infecção ativa na ocasião, permitindo, assim, o implante de um material protético.

\section{CONCLUSÃO}

Os pseudoaneurismas de etiologia infecciosa são raros. O tratamento de escolha, na maioria dos casos, é a ressecção associada à revascularização do território perfundido por esse vaso. Pseudoaneurismas micóticos de apresentação tardia sem infecção ativa podem ser manejados com técnicas endovasculares, principalmente nos casos de localizações atípicas ou de acesso cirúrgico mais restrito.

\section{REFERÊNCIAS}

1. Osler W. Malignant endocarditis. Br Med J. 1885;1(1262):467-70. http://dx.doi.org/10.1136/bmj.1.1262.467. PMid:20751186.

2. McKee MA, Ballard JL. Mycotic aneurysms of the tibioperoneal arteries. Ann Vasc Surg. 1999;13(2):188-90. http://dx.doi.org/10.1007/ s100169900240. PMid: 10072460.

3. Belczak SQ, Sincos IR, Teivelis MP, Oliveira CAS, Fragoso H, Aun R. Mycotic aneurysm of the tibioperoneal trunk: a first manifestation of an infected endocarditis. Rev Inst Med Trop Sao Paulo. 2012;54(3):171-4. http://dx.doi.org/10.1590/S003646652012000300010. PMid:22634890.

4. Barros MVL, Penna JTM, Henriques JF, Roquette-Reis F, Labropoulos $\mathrm{N}$. Infrapopliteal mycotic aneurysm caused by endocarditis - case report and literature review. Echocardiography. 2010;27(1):77-9. http://dx.doi.org/10.1111/j.1540-8175.2009.01008.x. PMid:20380664.

5. Khasnis A, Chick D, Havlichek D Jr. Mycotic aneurysm of the tibioperoneal trunk as a complication of aortic valve endocarditis due to lactobacillus casei infection : case report and review of literature. Infect Dis Clin Pract. 2006;14(3):185-7. http://dx.doi. org/10.1097/01.idc.0000161506.18631.f4.
6. Akers DL Jr, Fowl RJ, Kempczinski RF. Mycotic aneurysm of the tibioperoneal trunk: case report and review of the literature. J Vasc Surg. 1992;16(1):71-4. http://dx.doi.org/10.1016/07415214(92)90420-D. PMid:1619727.

7. Larena-Avellaneda A, Debus ES, Daum H, Kindel M, Gross-Fengels WIH, Imig H. Mycotic aneurysms affecting both lower legs of a patient with Candida endocarditis - endovascular therapy and open vascular surgery. Ann Vasc Surg. 2004;18(1):130-3. http:// dx.doi.org/10.1007/s10016-003-0088-9. PMid:15043025.

8. Sadat U, See T, Cousins C, Hayes P, Gaunt M. Peroneal artery pseudoaneurysm - a case report and literature review. BMC Surg. 2007;7(4):2-4. PMid:17394650.

9. Geremia G, Haklin M, Brennecke L. Embolization of experimentally created aneurysms with intravascular stent devices. AJNR Am J Neuroradiol. 1994;15(7):1223-31. PMid:7976930.

\section{Correspondência \\ Marcio Miyamotto \\ Rua Francisco Juglair, 77/505, Mossunguê CEP 81200-230 - Curitiba (PR), Brasil Tel.: (41) 99961-0486 \\ E-mail:miyamotto@gmail.com}

Informações sobre os autores

MM - Cirurgião vascular chefe, Serviço de Cirurgia Vascular do Hospital Universitário Cajuru (HUC), Pontifícia Universidade Católica do Paraná (PUC-PR); Cirurgião vascular e endovascular, Serviço de Cirurgia Vascular Elias Abrão, Hospital Nossa Senhora das Graças; Tutor da Liga Acadêmica de Medicina Vascular, Hospital Universitário Cajuru (LAMEV); Diretor do Instituto VESSEL de Aperfeiçoamento Endovascular

GSM e TTF - Acadêmicas do curso de Medicina, Pontifícia Universidade Católica do Paraná (PUC-PR); Integrantes da Liga Acadêmica de Medicina Vascular, Hospital Universitário Cajuru, Pontifícia Universidade Católica do Paraná (LAMEV).

MSE - Acadêmico do curso de Medicina, Universidade Federal do Paraná (UFPR); Integrante da Liga Acadêmica, Medicina Vascular Hospital Universitário Cajuru da Pontifícia Universidade Católica do Paraná (LAMEV).

GCSN - Médica residente, Serviço de Cirurgia Vascular e Endovascular

Elias Abrão, Hospital Nossa Senhora das Graças (HNSG).

IGR - Cirurgião vascular chefe do Serviço de Cirurgia Vascular,

Hospital e Maternidade Sagrada Família, e ex-residente, Serviço de Cirurgia Vascular e Endovascular Elias Abrão, Hospital Nossa Senhora das Graças (HNSG)

CLR - Cirurgiã vascular, Serviço de Cirurgia Vascular, Hospital Universitário Cajuru (HUC), Pontifícia Universidade Católica do Paraná

(PUC-PR); cirurgião vascular, Instituto VESSEL de Aperfeiçoamento Endovascular

FRG - Cirurgiã vascular, Serviço de Cirurgia Vascular, Hospital Santa Cruz.

Contribuições dos autores Concepção e desenho do estudo: MM Análise e interpretação dos dados: MM

Coleta de dados: MM, GSM, TTF, MSE, GCSN, IGR, CLR, FRC Redação do artigo: MM, GSM, TTF, MSE, GCSN, IGR, CLR, FRG Revisão crítica do texto: $M M$ Aprovação final do artigo*: MM, GSM, TTF, MSE, GCSN, IGR, CLR,

Análise estatística: N/A Responsabilidade geral pelo estudo: MM

*Todos os autores leram e aprovaram a versão final submetida ao I Vasc Bras. 